\title{
THE OUTCOMES AND COSTS OF FALLS IN ELDERLY WOMEN
}

\author{
Inga Tamulaitytè-Morozovienė, Marija Tamulaitienè, Rimantas Stukas, Vidmantas Alekna
}

Faculty of Medicine of Vilnius University, Vilnius, Lithuania

\section{INTRODUCTION}

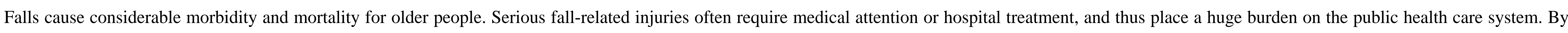

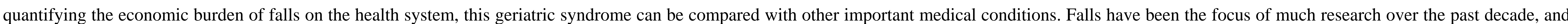
the annual cost burden in different countries has been estimated, mostly for fall-related fractures. There are no data on the cost of falls in Lithuania.

The aim of this study was to examine the outcomes and costs of direct medical care for falls in community-dwelling elderly women.

\section{METHODS}

Participants of this cross-sectional study were urban-living women aged 65 years and older who had consecutively visited the out-patient clinic National Osteoporosis Center for diagnostic or treatment procedures and who agreed to be interviewed by phone. The women who were independently mobile and cognitively competent were recruited. The local Ethics Committee approved the study protocol.

The interview was conducted by trained interviewers. Fall information was collected by standard questionnaire specially prepared for this study according to scientific studies on falls and their consequences and containing 28 questions. The interview questionnaire included six sections covering socio-demographic characteristics, health status and medication used before falling, number of falls, consequences of each fall, and health care procedures obtained because of falls.

A self-report of one or more falls within in the past year was obtained. Respondents were asked to describe the reason and location of every fall and to describe all the injuries they have sustained because of falling. Then participants were asked about the contacts with primary and secondary care medical

\section{RESULTS}

The study population consisted of 878 community-dwelling women, aged $65-90$ years. Falls during the previous 12 months were reported by 310 (35.3\%) women. Overall, the details of 407 falls were recorded. Among all fallers, $65.4 \%$ reported a single fall, $20.6 \%$ fell twice, and $14 \%$ sustained three or more falls. The mean age of respondents was $72.2 \pm 4.8$ years (65-90 years), the age of 647 women was 65-74 years and 231 women were aged 75-90. There was no statistical difference in the mean age of those who fell and who did not $(72.7 \pm 5$ and $72.0 \pm 4.7$ years, respectively; $p=0.04)$. Women over 75 years fell more frequently than younger $(\mathrm{p}=0.021)$.

Outcomes

Of all women who fell, 280 (90.3\%) reported their fall resulted in an injury, and total of 331 fall were injurious. The vast majority of fall-related injuries were contusions, skin abrasions, or fractures (Table 1).

Table 1. The frequency of fall-related consequences

\begin{tabular}{lcc}
\hline \multirow{2}{*}{ Type of injury } & \multicolumn{2}{c}{ Number of injuries } \\
\cline { 2 - 3 } & $\mathbf{n}$ & \% \\
\hline Contusions & 250 & 58.3 \\
Abrasions & 32 & 7.5 \\
Back pain & 26 & 6.1 \\
Open wounds & 15 & 3.5 \\
Sprains & 9 & 2.1 \\
Joint dislocations & 5 & 1.2 \\
Skull/brain injuries & 15 & 3.5 \\
Fractures & 77 & 17.9 \\
\hline Total & 429 & 100 \\
Other outcomes: & $\mathrm{n}$ & $\%$ of fallers \\
Fear of falling & 226 & 72.9 \\
Restricted daily activities & 124 & 40.0 \\
Use of pain relievers & 125 & 40.3 \\
\hline
\end{tabular}

Seventy seven participants (24.8\% of all fallers, and $27.5 \%$ of those injured) reported they have sustained a bone fracture related to fall. Fractures comprised $17.9 \%$ of all injuries. Among fractures reported, $53.2 \%$ were forearm fractures, $7.8 \%$ were hip, $9.1 \%$ - vertebral, and $29.9 \%$ - other fractures

Fear of falling was statistically significantly more frequent in those women who fractured comparing to those who did not $(90.9 \%$ and $67 \%$, respectively). The same was observed when the frequency of restricted daily activities was analyzed: this was reported by $63.6 \%$ of women who had sustained fallrelated fractures and by $36.4 \%$ those who had not $(\mathrm{p}<0.0001)$, and mostly was explained by fair of falling. More than third of respondents who fell, reported they have started to use additional medications, and this mostly were pain medications.

\section{CONCLUSIONS}

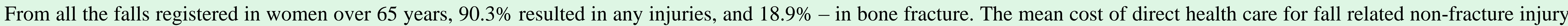
was 194 EUR. The costs for fall with hip fracture were the highest and reached 2571 EUR.

\section{ACKNOWLEDGMENTS}

We are grateful to National Osteoporosis Center for the possibility to conduct this study and to its staff for contribution to the survey. services: how many times they had seen a family doctor or consultant. Those participants who had been hospitalized for the consequences of fall were also asked about their length of stay in the orthopaedic and/or rehabilitation department at hospital. Finally, women were asked to describe any health care procedures performed at out-patient clinic or emergency department and medications that had been prescribed specifically for their fall.

The cost of health care due to fall was estimated after calculating the sum of costs for all out-patient visits, procedures or hospitalizations, excluding the cost of medication and medical equipment. The total costs of fall-related care were calculated as the weighted sum of costs for all fall-related medical events aggregated across all fallers. Mean costs per faller and per faller receiving medical care were calculated. The statistical analysis was performed using SPSS software for Windows (version 18.0). All p-values less than 0.05 were considered as statistically significant.

\section{Health care and costs}

Fall-related medical care during the previous 12 months was provided to 115 individuals (37\% of those fallen, and $41 \%$ of those injured in fall). The highest proportion of respondents reported the use of outpatient medical services. Numbers of out-patient visits ranged from 1 to 13, mostly from 2 to 4 visits were reported. The mean numbers of health care procedures by type are shown in Table 2 .

Table 2. Mean number of out-patient visits due to falls

\begin{tabular}{lccc}
\hline \multicolumn{1}{c}{ Specialist seen: } & Number of subjects & Number of visits & $\begin{array}{c}\text { Mean number of visits per faller } \\
(\mathbf{9 5} \% \mathbf{C I})\end{array}$ \\
\hline Family doctor & 60 & 114 & $0.37(0.26-0.48)$ \\
Traumatologist & 54 & 154 & $0.50(0.35-0.65)$ \\
Neurologist & 5 & 9 & $0.03(0.00-0.06)$ \\
Surgeon & 48 & 140 & $0.45(0.30-0.60)$ \\
Radiologist & 97 & 112 & $0.36(0.24-0.49)$ \\
Other & 4 & 6 & $0.02(0.00-0.04)$ \\
Total & 115 & 535 & $1.73(1.36-2.10)$ \\
\hline
\end{tabular}

The majority of specialists visited were traumatologist, surgeon, and radiologist. Ambulance was used by $11.9 \%$, and hospitalization - by $4.8 \%$ of women who fell. The percentages of women visiting the outpatient facility, as well as the mean number of health care procedures was higher in women with fallrelated fractures when compared to non-fractured persons ( $p<0,0001)$. As many as 15 women $(4.8 \%$ of all fallen subjects and $11.1 \%$ of those who sought medical attention for a fall) were hospitalized to orthopaedic department for the treatment of fall-related injury, mostly because of fracture. The mean duration of hospitalization was $16.5 \pm 24.7$ days (ranged $3-90$ days). All the cases of hip fracture were treated at hospital while only $9.8 \%$ of those sustained forearm fracture were hospitalized, with a mean duration of $29.7 \pm 36.5 ; 5.8 \pm 5.5$ days, respectively. Only one of those 7 women sustained vertebral fracture was hospitalized for 12 days. In-patient rehabilitation was provided to 5 subjects with fractures, the mean duration of stay was $21.2 \pm 9.26$ days.

It was found that the mean total cost was 266.8 EUR per patient receiving the fall-related medical care. The largest part (almost a half) of this cost was caused by the out-patient visits and procedures. The mean estimated direct health care cost was 194 EUR for the fall with non-fracture injuries. When the costs were estimated in subjects who had sustained the fall-related fracture, higher numbers were found, with a significant difference in fractures of different location. The highest mean cost of health care (2571 EUR) was found in women with hip fractures, and more than a half $(52.1 \%)$ of it was comprised by in-patient hospitalization. The lowest cost per fall (219 EUR) fracture was in cases of forearm fracture. 